\title{
Dead space to tidal volume ratio (VD/VT) to explain extubation failure in children: the limitations of current evidence
}

\author{
Robinder G. Khemani*
}

Riou et al. ${ }^{1}$ present a manuscript describing the value of dead space to tidal volume ratio (VD/VT) to evaluate the likelihood of successful extubation from mechanical ventilation in children at the intensive care unit (ICU). The study methods mimic two papers previously published on the same topic regarding critically ill children.2,3 Like the study performed by Hubble et al., ${ }^{2}$ Riou et al. found that VD/VT > 0.55, calculated shortly before extubation, while the patient is breathing spontaneously with pressure support, has a strong association with extubation failure, presenting a positive likelihood ratio (LR) close to 4 (moderately high to rule in extubation failure), and negative $\mathrm{LR}<0.1$ (strongly negative to rule in extubation failure). ${ }^{1}$ VD/VT had good discrimination ability, with an area under the receiver operating characteristic curve (AUC) of 0.86 (95\% confidence interval [95\%CI] 0.73-0.98). ${ }^{1}$ This data contrast with a previous study by Bousso et al. which reported that VD/VTs $>0.65$, with a positive $\mathrm{LR}<2$, a negative $\mathrm{LR}=0.45$ and fair discrimination ability AUC $=0.621$ (95\%CI not reported), had poor association with extubation failure. ${ }^{3}$

So, how do we resolve the seemingly conflicting conclusions amongst these studies? While all three studies had similar methods regarding the conditions in which VD/ VTs measurements took place, significant differences in patient demographics, diagnoses, degree of parenchymal lung disease, tidal volume, and ICU specific practices regarding extubation with variable rates of extubation failure highlight the difficulty in expanding evidence generated from single institution studies with small sample sizes to other settings. This is particularly challenging for risk stratification studies in which the outcomes are somewhat subjective and institution-dependent (e.g., decision to implement noninvasive ventilation or reintubation).

Moreover, although the authors of all three studies describe standardized assessments for post extubation upper airway obstruction (UAO), none of these studies presents analysis stratified by this finding. Given that close to $1 / 3$ of all pediatric extubation failures are thought to be due to UAO on clinical grounds, ${ }^{4}$ and that there is no physiologic basis to surmise that VD/VT would predict the need for further respiratory support for UAO, differences in the performance of VD/VT amongst these three studies could be partially explained by UAO . Hubble et al. reported that four patients had clinician-judged UAO after extubation, three of whom needed further respiratory support. ${ }^{2}$ All three of these patients had VD/VTs $>0.55$, one presented atelectasis on chest X-ray, and one was later determined to have tracheobronchomalacia. ${ }^{2}$ Was their need for further respiratory therapy a result of residual lung disease, for which VD/VT would be expected to be elevated, or was it a consequence of UAO? The other two studies did not report the number of patients with clinician-judged UAO and their VD/VTs. Furthermore, clinician-judged UAO is subject to significant interobserver variability, 5,6 even with rigorous common training on stridor scores.

\footnotetext{
* MD, MsCl. Associate director of research, Department of Anesthesiology and Critical Care Medicine, Children's Hospital Los Angeles, Los Angeles, CA, USA. Assistant professor of pediatrics, Keck School of Medicine, University of Southern California, Los Angeles, CA, USA.

No conflicts of interest declared concerning the publication of this editorial.

Suggested citation: Khemani RG. Dead space to tidal volume ratio (VD/VT) to explain extubation failure in children: the limitations of current evidence. J Pediatr (Rio J). 2012;88(3):191-4.

http://dx.doi.org/10.2223/JPED.2195
} 
Notwithstanding UAO, significant differences amongst the patient populations in these studies may in part explain the variable performance of VD/VT in predicting successful extubation. The studies by Hubble et al. ${ }^{2}$ and Riou et al. ${ }^{1}$ were performed in mixed medical-surgical ICUs. These studies had many perioperative patients, compared to only three in the study by Bousso et al. ${ }^{3}$ This highlights the importance of patient mix and also reasons for intubation in determining the predictive value of a marker of extubation readiness. Given the myriad of conditions that lead patients to require mechanical ventilation in pediatric ICUs, it is likely that a good portion of patients in these two studies had relatively healthy lungs (38\% in Riou et al., ${ }^{1}$ and $53 \%$ in Hubble et al. ${ }^{2}$ ), with the reason for intubation being airway protection (e.g., in the case of status epilepticus, coma, trauma, airway abnormalities), or post-operative sedation. The study by Bousso et al. had 16 patients with neurologic conditions, but approximately $75 \%$ of their patients had a diagnosis consistent with lung or lower airways disease. ${ }^{3}$ Given that VD/VT is a marker of lung disease severity, and studies evaluating its association with objective outcomes such as mortality have been made with children and adults with hypoxemic respiratory failure, acute lung injury, 7,8 or congenital diaphragmatic hernia, ${ }^{9}$ evidences show that patients with lung disease (in contrast to upper airway or central nervous system disease) have more abnormal VD/ VT. While a recent study has determined that elevated VD/VT in the first post-operative day is associated with prolonged mechanical ventilation in patients undergoing surgery for congenital heart disease, ${ }^{10}$ elevations in VD/VT in this group are likely more related to issues with abnormal cardiac output.

When performing extubation readiness testing, clinicians not only assess residual pulmonary disease, of which VD/ VT is a marker, during a spontaneous breathing trial; but they also assess sedation, airway protection, and neuromuscular strength. ${ }^{11}$ These latter measures are very important to determine when patients intubated for airway protection or post-operative sedation are ready for extubation. Given that these patients were not intubated for lung disease, they would not be expected to have elevations in VD/VT. Therefore, their likelihood for successful extubation is dependent on the clinician's assessment of sedation, airway protection, and disease resolution (e.g., seizure is over). Correct assessment of these conditions is generally more straightforward than assessing residual lung disease, so the likelihood for extubation failure or noninvasive ventilation after extubation is very low for that group of patients. In contrast, the four patients who failed extubation in the study by Riou et al. had septic shock, pneumonia, acute respiratory distress syndrome, and Duchenne muscular dystrophy. ${ }^{1}$ These diagnoses are consistent with patients who could have residual lung disease near extubation, and VD/VT has a physiologic basis to potentially quantify the severity of it. However, mixing together patients with no lung disease (and, therefore, normal VD/VT) who have a low risk for additional support after extubation since it is easier to assess the other components of extubation readiness, with patients with lung disease (and potentially abnormal VD/VT) in whom disease resolution is harder to assess, will artificially raise the predictive value of VD/VT. This may be part of the reason why the study by Bousso et al. found no difference in VD/VT amongst those who had successful extubation and those who had unsuccessful extubation, since close to $75 \%$ of patients were intubated for lung or lower airway disease. ${ }^{3}$

The study by Bousso et al., ${ }^{3}$ however, may have underestimated the predictive ability of VD/VT because of the choice of delivered tidal volume. Given that anatomic dead space is relatively fixed, delivering a relatively small tidal volume breath will result in a larger fraction of the volume being delivered to the anatomic dead space than with a larger tidal volume breath. This may result in a measured VD/VT lower than the one which would be observed with a larger breath. More importantly, the degree of alveolar dead space changes as a function of lung recruitment and also over distention. As such, the choice of delivered (or targeted) tidal volume is important in evaluating VD/VT measurements. The studies by Hubble et al. and Riou et al. had mean tidal volume between $6.4-8.3 \mathrm{~mL} / \mathrm{kg}^{1,2}$ in contrast to the study by Bousso et al. which presented mean tidal volume of $53 \mathrm{~mL}$ (by extrapolation, 4.8-6.1 mL/ $\mathrm{kg}){ }^{3}$ This, in conjunction with the higher percentage of children with parenchymal lung disease, may explain why the mean VD/VT was much higher in the study by Bousso et al. (mean $=0.62-0.65)^{3}$ compared to the other two studies (mean $=0.41-0.59$ and 0.44-0.68).1,2 The study by Bousso et al. also had younger patients (mean age $=17$ months, median age $=6$ months) ${ }^{3}$ compared to the other two studies (mean age $=4-6$ years). ${ }^{1,2}$ These younger patients likely had a more rapid shallow breathing pattern with smaller tidal volumes, consequently. More importantly, the performance of VD/VT as a predictor of extubation success may be different in young children as an effect modification. Bousso et al. describe alternate risk factors for younger infants: less resistance to muscle fatigue, less effective cough to clear secretions, and proportionally narrow airways. ${ }^{3}$ If these or other risk factors for extubation failure unique to younger children are not captured by VD/VT, then its performance as a predictive marker will be inferior.

Finally, the different results in these studies emphasize how the incidence of the outcome variable (respiratory support after extubation) can affect the results. In the study by Riou et al., less than $10 \%$ of patients received respiratory support after extubation, ${ }^{1} 20 \%$ received it in the study by Hubble et al. (9/45), ${ }^{2}$ and $24 \%$ received it in the study by Bousso et al. (21/86). ${ }^{3}$ None of the 
patients in Riou et al.'s study were reintubated, ${ }^{1}$ while in the study by Hubble et al. three patients $(6.7 \%)$ were reintubated ${ }^{2}$ and 10 patients $(11.7 \%)$ in Bousso et al.'s study. ${ }^{3}$ Unfortunately, these outcomes are not objective, since a practitioner's decision to implement further respiratory support may vary amongst institutions. This, along with case mix within ICUs, may explain the difference in the prevalence of outcomes. Given that sensitivity, specificity, positive and negative predictive values can be affected by disease prevalence, it is important to analyze the results in relation to LRs and to generated post-test probabilities. The studies by Riou et al. and Hubble et al. presented positive LRs close to 4 and negative LRs $<0.1 .1,2$ Given the high incidence of children without parenchymal disease, this may be an overestimation of the performance of the VD/VT cutoff of 0.5 or 0.55 . In contrast, the positive LR of 1.9 and negative LR of 0.65 in the study by Bousso et al. ${ }^{3}$ may be an underestimation of how well a VD/VT cutoff of 0.65 may perform in older children or in those with higher tidal volumes. So, when attempting to generalize this to different ICUs, practitioners should be cognizant of their own extubation failure rate, the risks of additional days of invasive mechanical ventilation compared to the risks of noninvasive mechanical ventilation, and also of which extubation failure rate they will tolerate. If one takes a positive LR of 3 , with a pretest probability of $10 \%$, then the likelihood of extubation failure based on VD/VT is of $25 \%$. Most would say this figure is too high. In contrast, if the negative LR is 0.5 , with a pretest probability of $10 \%$, then the likelihood of extubation failure is $5 \%$, which most would call acceptable. One should make these calculations based upon their local extubation failure rate, and their target extubation failure rate.

Given the relatively small number of outcomes from these studies, the confidence in the performance of VD/VT in predicting extubation failure is not high. Although not reported in the papers, the $95 \% \mathrm{CI}$ for positive LR in the study by Hubble et al. ranges from 1.99 to 6.87 , with a negative LR ranging from 0.015 to $0.68 .^{2}$ Given that no patient was reintubated in the study by Riou et al., ${ }^{1}$ one has to estimate the negative LR, which puts the $95 \% \mathrm{CI}$ for the positive LR between 1.87 and 7.30, and the negative LR between 0.01 and 2.20. Unfortunately, Bousso et al. do not report raw data to calculate $95 \%$ CIs. ${ }^{3}$ However, the $95 \%$ CIs from the two positive studies ${ }^{1,2}$ contain the point estimates for positive and negative LRs reported in the Bousso et al.'s study. ${ }^{3}$ Therefore, the small sample sizes of these studies greatly limit their generalizability and do not support definitive conclusions regarding the value or optimal cutoff of VD/VT in predicting extubation success.

While it is clear that we need a large-scale multicentered trial to increase sample size and precision in estimates of performance of VD/VT on extubation success, we must pay attention to the study design. As these papers have highlighted, a multicenter study must address the subjective nature of the outcome of respiratory support after ventilation through some standardized criteria for application of noninvasive support or reintubation within ICUs. It must also deal with UAO after extubation in a standardized fashion, overcoming the interrater variability in the clinical assessment of stridor. Furthermore, the study must have an appropriate case mix by ensuring an adequate number of children with parenchymal lung disease in whom the VD/VT has physiologic basis for predicting outcome. It must appropriately standardize tidal volume to ensure accurate and reproducible calculation of VD/VT, and, finally, it should be powered to evaluate particular subgroups of patients, based on demographics such as age. Until then, a pragmatic approach may be to capitalize on the negative LR of VD/VT $<0.5$. Then, if patients have a low VD/VT $(<0.5)$, assuming they pass other elements of extubation readiness, the likelihood of need for further respiratory therapy after extubation is low. However, if patients have elevated VD/VT ( $>0.5$ ), it is unclear how much more at risk they may be for extubation failure.

\section{References}

1. Riou Y, Chaari W, Leteurtre S, Leclerc F. Predictive value of the physiologic deadspace/tidal volume ratio in the weaning process of mechanical ventilation in children. J Pediatr (Rio J). 2012;88:217-21

2. Hubble CL, Gentile MA, Tripp DS, Craig DM, Meliones JN, Cheifetz IM. Deadspace to tidal volume ratio predicts successful extubation in infants and children. Crit Care Med. 2000;28:2034-40.

3. Bousso A, Ejzenberg B, Ventura AM, Fernandes JC, Fernandes IC, Góes PF, et al. Evaluation of the dead space to tidal volume ratio as a predictor of extubation failure. J Pediatr (Rio J). $2006 ; 82: 347-53$

4. Kurachek SC, Newth CJ, Quasney MW, Rice T, Sachdeva RC, Patel NR, et al. Extubation failure in pediatric intensive care: a multiple-center study of risk factors and outcomes. Crit Care Med. 2003;31:2657-64.

5. Khemani RG. Post extubation stridor the call for objectivity. Indian Pediatr. 2010;47:307-8.

6. Kemper $\mathrm{KJ}$, Benson MS, Bishop MJ. Interobserver variability in assessing pediatric postextubation stridor. Clin Pediatr (Phila). $1992 ; 31: 405-8$

7. Ghuman AK, Newth CJ, Khemani RG. The association between the end tidal alveolar dead space fraction and mortality in pediatric acute hypoxemic respiratory failure. Pediatr Crit Care Med. 2012;13:11-5.

8. Raurich JM, Vilar M, Colomar A, Ibáñez J, Ayestarán I, Pérez-Bárcena J, et al. Prognostic value of the pulmonary dead-space fraction during the early and intermediate phases of acute respiratory distress syndrome. Respir Care. 2010;55:282-7.

9. Arnold JH, Bower LK, Thompson JE. Respiratory deadspace measurements in neonates with congenital diaphragmatic hernia. Crit Care Med. 1995;23:371-5. 
10. Ong T, Stuart-Killion RB, Daniel BM, Presnell LB, Zhuo H, Matthay $M A$, et al. Higher pulmonary dead space may predict prolonged mechanical ventilation after cardiac surgery. Pediatr Pulmonol. 2009;44:457-63.

11. Newth CJ, Venkataraman S, Willson DF, Meert KL, Harrison R, Dean JM, et al. Weaning and extubation readiness in pediatric patients. Pediatr Crit Care Med. 2009;10:1-11.
Correspondence:

Robinder G. Khemani

Dept. of Anesthesiology and Critical Care Medicine, Children's Hospital Los Angeles

4650 Sunset Blvd., Mailstop n.12

90027 - Los Angeles, CA - USA

Tel.: +1 (323) 361.2376

Fax: +1 (323) 361.1001

E-mail: rkhemani@chla.usc.edu 